\title{
Analyzing Medication Documentation in Electronic Health Records: Dental Students' Self-Reported Behaviors and Charting Practices
}

\author{
Wesley K. Burcham, Laura M. Romito, Elizabeth A. Moser, Bruce D. Gitter
}

Abstract: The aim of this two-part study was to assess third- and fourth-year dental students' perceptions, self-reported behaviors, and actual charting practices regarding medication documentation in axiUm, the electronic health record (EHR) system. In part one of the study, in fall 2015, all 125 third- and 85 fourth-year dental students at one U.S. dental school were invited to complete a ten-item anonymous survey on medication history-taking. In part two of the study, the EHRs of 519 recent dental school patients were randomly chosen via axiUm query based on age $>21$ years and the presence of at least one documented medication. Documentation completeness was assessed per EHR and each medication based on proper medication name, classification, dose/ frequency, indication, potential oral effects, and correct medication spelling. Consistency was evaluated by identifying the presence/absence of a medical reason for each medication. The survey response rate was $90.6 \%(\mathrm{~N}=187)$. In total, $64.5 \%$ of responding students reported that taking a complete medication history is important and useful in enhancing pharmacology knowledge; $90.4 \%$ perceived it helped improve their understanding of patients' medical conditions. The fourth-year students were more likely than the third-year students to value the latter $(\mathrm{p}=0.0236$ ). Overall, $48.6 \%$ reported reviewing patient medications with clinic faculty $76-100 \%$ of the time. The respondents' most frequently cited perceived barriers to medication documentation were patients' not knowing their medications $(68.5 \%)$ and, to a much lesser degree, axiUm limitations (14\%). Proper medication name was most often recorded $(93.6 \%)$, and potential oral effects were recorded the least (3.0\%). Medication/medical condition consistency was $70.6 \%$. In this study, most of the students perceived patient medication documentation as important; however, many did not appreciate the importance of all elements of a complete medication history, and complete medication documentation was low.

Wesley K. Burcham, DDS, is a 2018 graduate of Indiana University School of Dentistry; Laura M. Romito, DDS, MS, MBA, is Associate Professor, Department of Biomedical Sciences and Comprehensive Care, Indiana University School of Dentistry; Elizabeth A. Moser, MS, is a biostatistician, Department of Biostatistics, Indiana University School of Medicine and Richard M. Fairbanks School of Public Health; and Bruce D. Gitter, PhD, is Clinical Professor, Department of Biomedical Sciences and Comprehensive Care, Indiana University School of Dentistry. Direct correspondence to Dr. Laura M. Romito, Indiana University School of Dentistry, Rm. 243A, 1121 W. Michigan St., Indianapolis, IN 46202; 317-278-6210; 1romitoc@iu.edu.

Keywords: dental education, dental students, medication, medication reconciliation, pharmacology, electronic health record

Submitted for publication 6/27/18; accepted 11/8/18; first published online 3/25/19

doi: $10.21815 / J D E .019 .070$

W Then dental patients present with complex medical histories, including multiple chronic illnesses managed by a variety of medications, the extent to which medications affect their systemic health, oral health, and dental management is significant. As such, procurement of a thorough medication history, as a component of the medical history, before rendering dental treatment is the standard of care and an essential component of ethical practice. ${ }^{1,2}$

According to the Centers for Disease Control and Prevention (CDC), in any one month from 2011 through $2014,48.9 \%$ of all Americans took at least one prescription drug, $23.1 \%$ took three or four medications, and $11.9 \%$ used five or more. ${ }^{3}$ Polypharmacy increases the risk of adverse events, of which $5-10 \%$ are due to drug interactions, and these interactions can result in medication toxicity, loss of drug efficacy, or unexpected effects. ${ }^{4}$ Medically compromised patients may be at greater risk for adverse events due to polypharmacy compounded by the diminished physiologic reserve associated with several co-morbidities. Thus, the dentist must avoid administering/prescribing medications that may interact with the patient's current medications. Dentists with an understanding of patients' medications can also educate them about the potential oral effects of the medications being prescribed. For example, there are over 200 drugs alone that can alter taste and over 500 that have xerostomia as a reported side effect. ${ }^{5}$

In an academic setting, an accurate medication history, in conjunction with the medical history, also 
serves as an important tool for reinforcing dental students' pharmacological and biomedical science knowledge and integrating this knowledge into safe clinical care. In many dental curricula, pharmacology instruction is limited to an early didactic course. More recently, dental schools have reported using multiple methodologies (such as problem-based learning, clinical cases, and online courses) to increase students' understanding and appreciation of the clinical relevance of pharmacology concepts and drug information. ${ }^{6-8}$

Along with didactic pharmacology instruction, the clinical experience of obtaining and documenting patient medication information as part of a comprehensive medical history provides an opportunity for students to apply pharmacology concepts to enhance their understanding of disease states, patients' overall health status, and the potential of medications to affect oral health and dental treatment. However, students may not be maximizing this opportunity. In a prior study with third-year dental students, pretestposttest assessment data were collected to evaluate a clinical pharmacology educational intervention using EHRs. ${ }^{9}$ Results from that study were that students whose patients reported taking more medications had increased pharmacology knowledge. Additionally, posttest scores increased when students documented the medication details rather than simply listing the medication name, and repetition in EHR documentation of medications correlated with increased student knowledge.

The literature is sparse on medication documentation as a component of the patient medical history transcribed by dental students. Anecdotal evidence suggests that student documentation of patient medications is neither comprehensive nor routinely aligned with other elements of the medical history. Further, no previous documented research exists in dental or allied health fields to determine the value and importance students place on obtaining complete and accurate medication histories or on their perceived barriers to comprehensively recording patient medications. Our study sought to address these gaps in the literature.

The aim of this two-part study was to assess third- and fourth-year dental students' perceptions, self-reported behaviors, and actual charting practices regarding medication documentation in axiUm, the electronic health record (EHR) system. Specifically, we sought to determine the degree to which the students perceived patients' medication history to be useful in understanding medical status; students' perceived barriers to completing documentation of patient medications; the level of completeness of the medication history in axiUm EHR (Exan Corporation, Vancouver, Canada); and the consistency between medications listed and patients' medical conditions as recorded by students in the EHR. We hypothesized that students' perceived value of comprehensive documentation of medications would be low, that their primary barrier to medication documentation would be lack of time, and that less than $30 \%$ of axiUm charts would have complete documentation of patient medications and show consistency between medications listed and patient medical conditions. The results of the study will inform modifications in curriculum, instruction, and clinical processes to optimize medication documentation in the EHR and increase students' appreciation for and integration of patients' medication information into their understanding of pharmacology and patients' medical conditions.

\section{Methods}

The Indiana University Institutional Review Board approved this study (\#1503218727). This crosssectional study was conducted with third-year (D3) and fourth-year (D4) dental students at Indiana University School of Dentistry (IUSD) in two parts in 2015.

\section{Part 1}

A ten-item survey instrument was developed by the investigators and peer-reviewed by faculty from the IUSD Research Committee. Subsequently, the survey was piloted with ten D4 students (Class of 2015) and revised based on pilot data. The survey had five Likert-scale items (Q1-Q5), two open-ended questions (Q7 and Q9), and three multiple-choice questions (Q6, Q8, and Q10). The survey assessed students' perceptions of the utility of a complete patient medication history; the frequency with which they obtained a complete medication history; perceived barriers to documenting patients' medications; resources used to obtain detailed drug information in clinic; and perceptions of what constitutes a complete drug citation in the medication history.

In fall 2015, numbered copies of the voluntary, anonymous paper survey, color-coded for each class, were distributed to all 125 D3 (Class of 2017) and 85 D4 (Class of 2016) students during each cohort's assembly. The study was explained, and students were given time to complete the survey. All surveys 
were collected, and data were separated by cohort, reviewed, coded, and entered into an electronic database for statistical analysis, which included counts, percentages, and descriptive statistics. Cohort comparisons on the Likert scale and multiple-choice items were evaluated using chi-square or Fisher's Exact tests and Wilcoxon Rank Sum tests. Responses to the two open-ended items were counted and grouped into themes for analysis.

\section{Part 2}

Following a power analysis indicating at least 402 records would provide a $95 \% \pm 5 \%$ confidence interval of our hypothesized value of $25-35 \%$, a total of 519 EHRs of patients seen in the school's comprehensive care (predoctoral) clinics from June 1, 2013, to June 2, 2015, were randomly selected for review from an axiUm query. Selections were based on two criteria: patient age $>21$ years and the presence of at least one documented medication. EHRs were oversampled to allow for the exclusion of those with unusable or missing data; a total of 489 EHRs were included in the final analysis. Only the EHR medical history data were extracted; we were provided with a usable, password-protected file of de-identified information for coding and analysis. For each EHR, the following axiUm medical history data were included: review of systems, current medications, past surgical history, allergies, social history, pregnancy status, blood pressure, pulse, and medical summary. Nearly all information in these sections obtained by dental students during patient history-taking and entered into axiUm was in the form of free text.

To determine the completeness of drug documentation in each EHR, the following six coding categories were applied to each medication listed in each EHR: proper medication name, correct medication spelling, classification, dose/frequency, indication, and potential oral effects. Each category was coded as a dichotomous variable: present (1) or not present (0) with the exception of dose/frequency. Dose/frequency was coded as 0 if neither dose nor frequency was documented, 1 if dose or frequency was documented, and 2 if both dose and frequency were documented. Completeness of medication documentation was assessed by scoring across the six categories. The range of possible total scores across all coded completeness categories was 0-7.

"Proper medication name" was defined as the specific generic or trade name that could be identified in a drug formulary. If a listed medication name-for example, ramipril-was misspelled but was discernible enough to be located in a drug reference source, then it was scored 1 for proper medication name but 0 in the medication spelling category. For example, to be considered correct (scored 1) in the proper medication name category, ramipril could be listed as "ramapril" but not "ramel" or "blood pressure pill."

In each EHR, the completeness of medication documentation was assessed for each drug (medication level) as well as for all medications as a group (patient level). "Medication level completeness" was defined as complete documentation (score of 7) of all six coding categories for each specific medication listed in an EHR. "Patient level completeness" was defined as complete documentation for all six coding categories (score of 7) for all medications identified in a patient's medical history. The presence of medical condition-medication consistency, defined as a medical condition indicated for each medication listed in an EHR, was coded as a dichotomous variable: consistency present (1) or not present (0). This study did not assess for consistency between a listed medical condition and the presence or lack of a medication as there may be multiple reasons for which a patient may not be taking a medication for a listed medical condition.

Scores for the completeness categories and consistency were recorded in a spreadsheet for each medication in each patient record. The medicationlevel \% completeness for each EHR was calculated using the aforementioned six components. The patientlevel \% completeness was calculated using the total of the score components for all medications recorded for each patient EHR. Categories that were not applicable to a particular medication were not included in calculation of the completeness percentage. Medication names were corrected and standardized where applicable. Frequencies for the score components, medication-medical condition consistency, and completeness categories were calculated, as well as basic statistics for completeness percentages for both medication-level and patient-level data. The percent completeness data were found to be normally distributed. One-way ANOVAs with a random effect for patient were used to compare medication-level $\%$ completeness for each of the individual scored components. One-way ANOVAs were used to compare patient-level $\%$ completeness. Chi-squared tests were conducted, and Odds Ratios were calculated for the agreement of medication-condition consistency and each of the record components (such as proper drug name and class). 


\section{Results}

\section{Part 1: Survey Response}

For the combined two cohorts, the survey response rate was $90.6 \%$ (total $\mathrm{N}=187$; D3 82.4\%, $\mathrm{N}=103$; D4 98.8\%, N=84). Some respondents skipped individual items. Overall, respondents were split as to whether the axiUm medication history intake format was an efficient way to comprehensively record patients' medications (Table 1). However, $87.4 \%$ of D3 respondents and $94 \%$ of D4 respondents either strongly agreed or agreed that the medication history aids in understanding patients' medical conditions. D4 respondents were more likely than D3 respondents to agree that medication history is an important tool to understanding patients' medical conditions $(\mathrm{p}=0.0236)$.

Of the total respondents to question $6(\mathrm{~N}=187)$, $48.6 \%$ reported reviewing patient medications with faculty during clinical encounters $76-100 \%$ of the

Table 1. Students' perceptions and self-reported behaviors regarding patients' medication documentation in axiUm electronic health records, by percentage of respondents to each item for D3 and D4 classes

\begin{tabular}{|c|c|c|c|c|c|c|c|c|}
\hline Survey Item & Class & $N$ & $\begin{array}{l}\text { Strongly } \\
\text { Disagree }\end{array}$ & Disagree & Agree & $\begin{array}{l}\text { Strongly } \\
\text { Agree }\end{array}$ & Mean (SD) & $\mathrm{p}$-value \\
\hline \multirow{2}{*}{$\begin{array}{l}\text { 1. I find the axiUm medication history } \\
\text { intake format to be an efficient way } \\
\text { to record patients' medications in a } \\
\text { comprehensive way. }\end{array}$} & D3 & 103 & $7.7 \%$ & $37.9 \%$ & $48.5 \%$ & $5.8 \%$ & $2.5(0.7)$ & \multirow[t]{2}{*}{0.4786} \\
\hline & D4 & 84 & $11.9 \%$ & $29.8 \%$ & $54.8 \%$ & $3.6 \%$ & $2.5(0.8)$ & \\
\hline \multirow{2}{*}{$\begin{array}{l}\text { 2. During my clinical encounters, all } \\
\text { of the patient's medications are always } \\
\text { reviewed with supervising faculty. }\end{array}$} & D3 & 103 & $5.8 \%$ & $38.8 \%$ & $39.8 \%$ & $15.5 \%$ & $2.7(0.8)$ & \multirow[t]{2}{*}{0.0962} \\
\hline & D4 & 84 & $6.0 \%$ & $50.0 \%$ & $39.3 \%$ & $4.8 \%$ & $2.4(0.7)$ & \\
\hline \multirow{2}{*}{$\begin{array}{l}\text { 3. The axiUm medication history } \\
\text { is an important tool in correctly } \\
\text { understanding the patient's medical } \\
\text { conditions. }\end{array}$} & D3 & 103 & $1.0 \%$ & $11.7 \%$ & $52.4 \%$ & $35.0 \%$ & $3.2(0.7)$ & \multirow[t]{2}{*}{0.0236} \\
\hline & D4 & 84 & 0 & $6.0 \%$ & $72.6 \%$ & $21.4 \%$ & $3.2(0.5)$ & \\
\hline \multirow{2}{*}{$\begin{array}{l}\text { 4. My patients' axiUm medication } \\
\text { history is a useful way for me to enhance } \\
\text { my pharmacology education. }\end{array}$} & D3 & 102 & $8.8 \%$ & $29.4 \%$ & $54.9 \%$ & $6.9 \%$ & $2.6(0.7)$ & \multirow[t]{2}{*}{0.3654} \\
\hline & D4 & 84 & $6.0 \%$ & $26.2 \%$ & $53.6 \%$ & $14.3 \%$ & $2.8(0.8)$ & \\
\hline \multirow{2}{*}{$\begin{array}{l}\text { 5. In clinical encounters, I often } \\
\text { consider the possibility that a patient's } \\
\text { medications may not be consistent with }\end{array}$} & D3 & 103 & 0 & $13.6 \%$ & $69.9 \%$ & $16.5 \%$ & $3.0(0.6)$ & \multirow[t]{2}{*}{0.1014} \\
\hline & D4 & 84 & $2.4 \%$ & $10.7 \%$ & $59.5 \%$ & $27.4 \%$ & $3.1(0.7)$ & \\
\hline
\end{tabular}
their reported medical conditions.

\begin{tabular}{|c|c|c|c|c|c|c|c|c|}
\hline & Class & $\mathrm{N}$ & $0-25 \%$ & $26-50 \%$ & $51-75 \%$ & $76-100 \%$ & Mean (SD) & p-value \\
\hline \multirow{2}{*}{$\begin{array}{l}\text { 6. During clinical encounters, my } \\
\text { patient's medication history is reviewed } \\
\text { with clinic faculty __ of the time. }\end{array}$} & D3 & 103 & $2.9 \%$ & $9.7 \%$ & $33.0 \%$ & $54.4 \%$ & $3.4(0.8)$ & \multirow[t]{3}{*}{0.0911} \\
\hline & D4 & 84 & 0 & $17.9 \%$ & $39.3 \%$ & $42.9 \%$ & $3.3(0.7)$ & \\
\hline 8. Sources of detailed drug information & Class & $N$ & No & Yes & p-value & & & \\
\hline \multirow{2}{*}{$\begin{array}{l}\text { I do not routinely look up or document } \\
\text { detailed information on a patient's } \\
\text { medications. }\end{array}$} & D3 & 99 & $88.9 \%$ & $11.1 \%$ & \multirow[t]{2}{*}{0.2468} & & & \\
\hline & D4 & 81 & $93.8 \%$ & $6.2 \%$ & & & & \\
\hline \multirow[t]{2}{*}{ Merck drug index } & D3 & 99 & $90.9 \%$ & $9.1 \%$ & \multirow[t]{2}{*}{0.1146} & & & \\
\hline & D4 & 81 & $97.5 \%$ & $2.5 \%$ & & & & \\
\hline \multirow[t]{2}{*}{ Lexicomp for dentistry } & D3 & 99 & $43.4 \%$ & $56.6 \%$ & \multirow[t]{2}{*}{0.7630} & & & \\
\hline & D4 & 81 & $45.7 \%$ & $54.3 \%$ & & & & \\
\hline \multirow[t]{2}{*}{ Micromedex } & D3 & 99 & $87.9 \%$ & $12.1 \%$ & \multirow[t]{2}{*}{0.0921} & & & \\
\hline & D4 & 81 & $95.1 \%$ & $4.9 \%$ & & & & \\
\hline \multirow[t]{2}{*}{ Mosby's drug reference } & D3 & 99 & $91.9 \%$ & $8.1 \%$ & \multirow[t]{2}{*}{0.3495} & & & \\
\hline & D4 & 81 & $96.3 \%$ & $3.7 \%$ & & & & \\
\hline \multirow{2}{*}{$\begin{array}{l}\text { Epocrates or similar app for smart phone } \\
\text { or tablet }\end{array}$} & D3 & 99 & $82.8 \%$ & $17.2 \%$ & \multirow{2}{*}{0.0121} & & & \\
\hline & D4 & 81 & $66.7 \%$ & $33.3 \%$ & & & & \\
\hline
\end{tabular}

Note: Question 8 was worded as follows: "During clinical encounters, from which of the following sources do you obtain detailed drug information to document in the patient's axiUm record? (Check all that apply)." 
time, and $36.1 \%$ reported doing so $51-75 \%$ of the time. Although overall there was no statistically significant difference between the cohorts $(\mathrm{p}=0.0911)$, a higher percentage of D3 than D4 students reported reviewing medication histories more than threequarters of the time with clinical faculty (Table 1). Overall, $91.1 \%$ of respondents to question $8(\mathrm{~N}=180)$ reported looking up information on their patients' medications. The most frequently cited resources were Lexicomp, Google, and Epocrates. Compared to the D3 students, the D4s were more likely to use Epocrates or a similar smartphone/tablet application as a drug information resource $(33.3 \%$ vs. $17.2 \% ; p=0.0121)$.

Question 10 asked students to indicate which items from a list of ten possible elements should be included in an EHR for a complete medication citation. In total, 178 responses were received: 97 from D3 and 81 from D4 students. Overall, the elements most frequently identified as necessary for a complete medication citation were the following: indication $(83.6 \%)$, dose $(79.8 \%)$, dose frequency $(78.7 \%)$, correct spelling (70.8\%), impact on oral health care $(65.2 \%)$, and proper generic or trade name $(59.0 \%)$. Figure 1 shows the responses by class. The association between student cohort and affirmative response that dose frequency was necessary for a complete medication citation was the only statistically significant response (D3 85.6\% vs. D4 70.4\%; $\mathrm{p}=0.0137$ ).

Responses from questions 7 and 9 were categorized into themes. Question 7 received 145 responses, with 81 from D3 and 64 from D4 students. Overall, the most frequently cited barriers to completing a comprehensive medication history for every patient were patients' not knowing/recalling their medication information (68.5\%), axiUm limitations (14\%), and difficulty understanding patient information/lack of pharmacology knowledge (4.5\%). Figure 2 shows responses by class. On the open-ended question 9, 224

\section{$100.00 \%$}

$90.00 \%$

$80.00 \%$

$70.00 \%$

$60.00 \%$

$50.00 \%$

$40.00 \%$

$30.00 \%$

$20.00 \%$

$10.00 \%$

$0.00 \%$
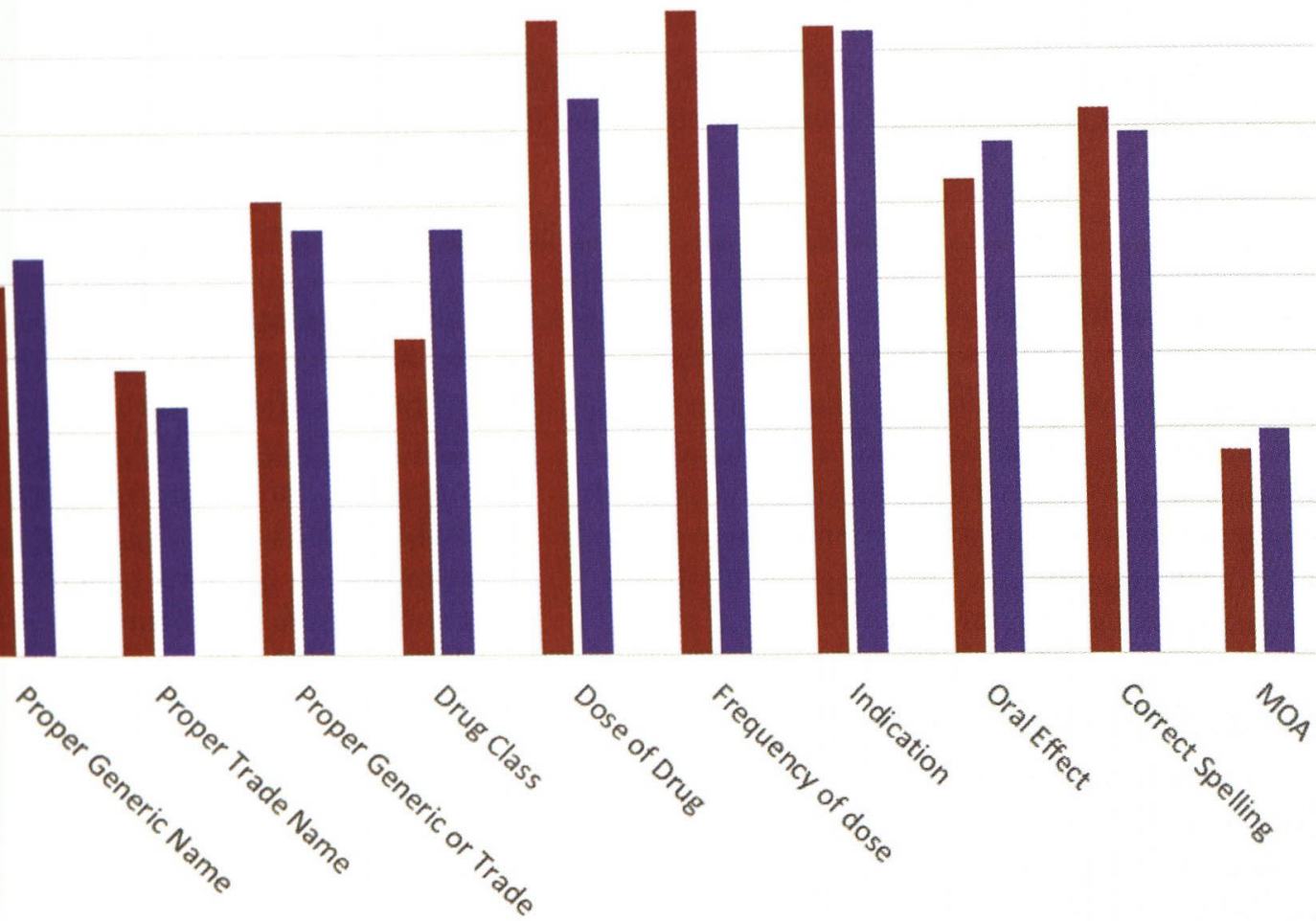

- 3rd Years

4th Years

Figure 1. Students' perceptions of what constitutes a complete drug citation in the axiUm medication history, by percentage of respondents in third year $(\mathrm{N}=97)$ and fourth year $(\mathrm{N}=81)$

$M O A=$ Mechanism of Action 


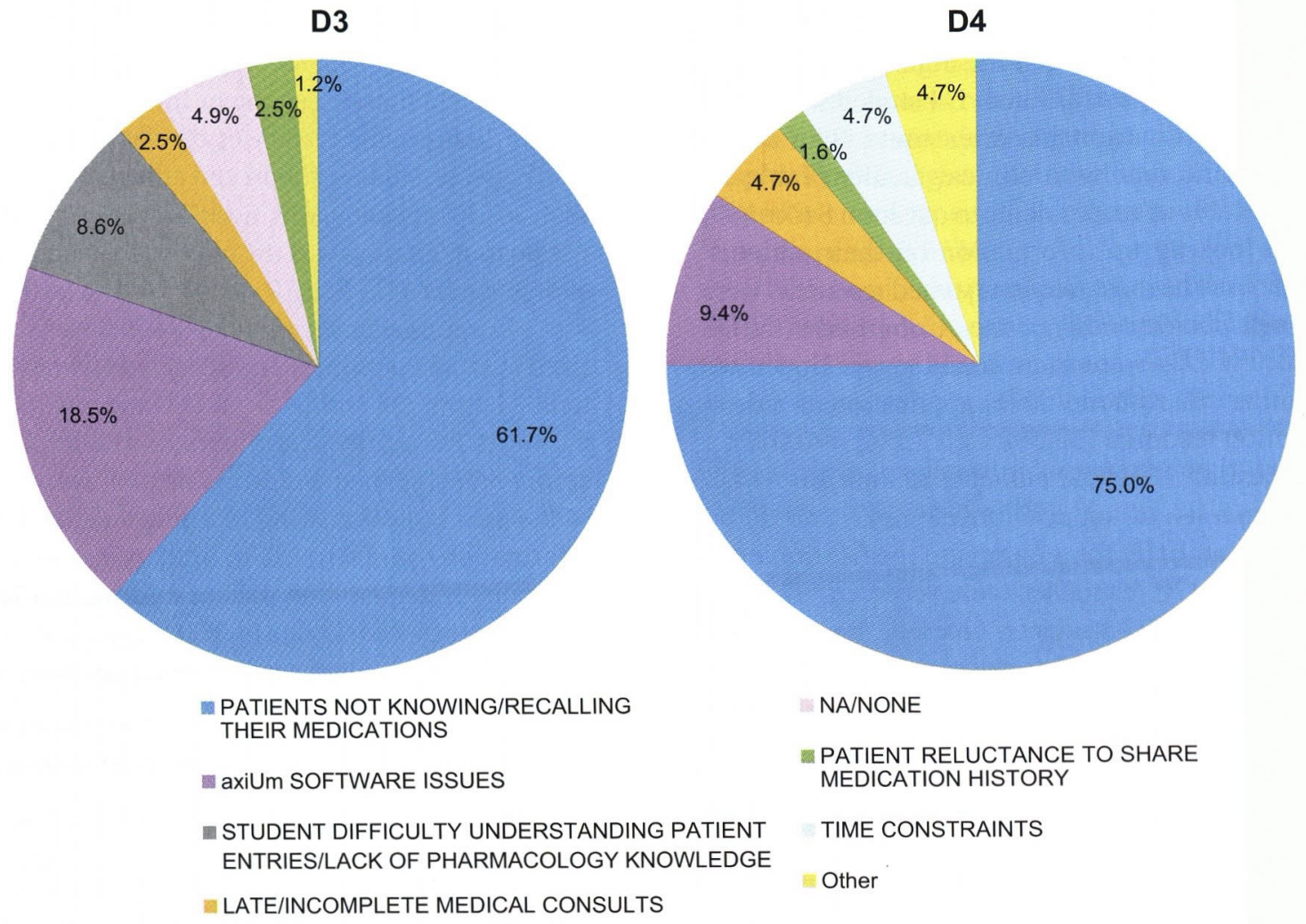

Figure 2. Students' reported barriers to complete documentation of patient medication history in axiUm electronic health record, by percentage of respondents in third year $(\mathrm{N}=81)$ and fourth year $(\mathrm{N}=64)$

$\mathrm{MOA}=$ Mechanism of Action

responses were received, with 128 from D3 and 96 from D4 students. The most frequently given reasons to accurately record medication history in the EHR were to understand how medications, interactions/ contraindications, and side effects can impact dental treatment/health (49\%); to understand patient health status/medical conditions (22.5\%); and to ensure/ promote patient safety $(16 \%)$.

\section{Part 2: EHR Analysis}

The medication information correctly recorded most frequently, in $93.6 \%$ of EHRs examined, was proper medication name. The information correctly recorded least frequently was potential oral effects $(3.0 \%)$ (Table 2). Overall, the consistency between documented medications and medical conditions was $70.6 \%$. The mean number of drugs recorded per patient was 3.9 (median=3).

Medication-level information was fully complete (having the maximum total score of 7 across all six medication coding categories) in $1.1 \%$ of records, and it was at least $70 \%$ complete in $13.7 \%$ of records (Table 3 ). However, for any given medication, an average of $45.7 \%$ of the information was complete. Patient-level medication information was fully complete in $0.2 \%$ of patients and at least $70 \%$ complete in $6.1 \%$ of patients. Patient-level record completeness had a mean of $44 \%$, with a standard deviation of $15.6 \%$ for the 489 patient charts. Thus, for any given EHR, an average of $44 \%$ of the medication information in that record was complete; this was significantly different from our hypothesized value of 30\% (Difference: 14\%; CI 12.6\%, 15.4\%; p<0.0001). Therefore, the overall frequency of complete patient medication information, albeit low, exceeded the hypothesized $30 \%$ level.

Correctly scoring an individual category was always associated with a higher medication-level \% completeness $(p<0.0001)$. EHRs with incorrectly recorded proper medication name had the lowest estimated $\%$ completeness (17.7\%; CI -31.33, -26.54; 
Table 2. Documentation in electronic health records, by number and percentage of records in each category receiving each score

\begin{tabular}{|c|c|c|c|c|}
\hline Category & $\begin{array}{c}\text { Number of } \\
\text { Records }\end{array}$ & Score $=0$ & Score $=1$ & Score $=2$ \\
\hline Proper drug name & 1,892 & $121(6.4 \%)$ & $1,771(93.6 \%)$ & $\mathrm{n} / \mathrm{a}$ \\
\hline Class & 1,883 & $1,727(91.7 \%)$ & $156(8.3 \%)$ & $\mathrm{n} / \mathrm{a}$ \\
\hline Dose/frequency & 1,892 & $807(42.7 \%)$ & $460(24.3 \%)$ & $625(33.0 \%)$ \\
\hline Indication & 1,892 & $1,028(54.3 \%)$ & $864(45.7 \%)$ & $\mathrm{n} / \mathrm{a}$ \\
\hline Potential oral effects & 1,892 & $1,835(97.0 \%)$ & $57(3.0 \%)$ & $\mathrm{n} / \mathrm{a}$ \\
\hline Medication spelling & 1,784 & $310(17.4 \%)$ & $1,474(82.6 \%)$ & $\mathrm{n} / \mathrm{a}$ \\
\hline Medication-condition consistency & 1,880 & $553(29.4 \%)$ & $1,327(70.6 \%)$ & $\mathrm{n} / \mathrm{a}$ \\
\hline
\end{tabular}

$\mathrm{n} / \mathrm{a}=$ not applicable for that category

Note: For six of the categories, a score of 0 meant not present, and a score of 1 meant present. Dose/frequency was coded 0 if neither dose nor frequency was documented, 1 if dose or frequency was documented, and 2 if both dose and frequency were documented.

Table 3. Documentation of complete medication in electronic health record at medication and patient levels, by number and percentage of records in each category

\begin{tabular}{lccc} 
Category & Number of Records & Yes & No \\
\hline Medication complete & 1,892 & $20(1.1 \%)$ & $1,872(98.9 \%)$ \\
Medication $\geq 70 \%$ complete & 1,892 & $260(13.7 \%)$ & $1,632(86.3 \%)$ \\
Patient complete & 489 & $1(0.2 \%)$ & $488(99.8 \%)$ \\
Patient $\geq 70 \%$ complete & 489 & $30(6.1 \%)$ & $459(93.9 \%)$ \\
\hline
\end{tabular}

$\mathrm{p}<0.0001)$, while those with recorded potential oral effects had the highest estimated \% completeness (74.8\%; CI -37.26, 24.99; p<0.0001). EHRs with medication-condition consistency also had a significantly, albeit modestly higher estimated medication $\%$ completeness than those that did not demonstrate consistency ( $46.0 \%$ vs. $41.1 \%$; $<<0.0001)$. The odds of having medication-condition consistency correct were $1.94(1.31,2.87)$ times higher if the student had recorded the proper drug name.

\section{Discussion}

This study assessed dental students' perceptions and self-reported behaviors regarding medication documentation in axiUm and, via an EHR review, evaluated the completeness of students' documentation of patient medications based on six elements of a medication history. These six components of the medication history make it more likely that students can detect inconsistencies with information obtained from the patient interview, including any inconsistencies between the medication list and patient-reported conditions (e.g., why does the patient report use of an antihypertensive medication but did not report hypertension?). In addition, medical errors resulting when one medication is mistaken for another can be avoided. Furthermore, proper documentation of patient medication history has a direct influence on students' understanding of patients' overall health status and implications for safe dental care.

While the vast majority of students in our study recognized the utility of the medication history in helping clinicians understand a patient's medical status, only about two-thirds saw this element as an opportunity to enhance their pharmacology knowledge (Table 1). It is possible that students viewed researching more detailed drug information and including that information in the EHR as unnecessary. However, the D4 students had a greater appreciation of the value of the medication history than did the D3 students, which is likely due to having more clinical experience and completing the D3 pharmacology course. Previous studies have found that greater appreciation of the value of the medication history in optimizing patient care and a better understanding of pharmacology were enhanced through dental-pharmacy interprofessional education experiences. ${ }^{10,11}$ 
In the survey findings, only about half of the students reviewed patient medications with faculty members in more than $75 \%$ of their clinical encounters. Furthermore, approximately $10 \%$ of the students stated that they did not routinely look up detailed information on their patient's medications at all (Table 1). The problem was highlighted in the discrepancy between students' self-reported survey responses and documentation in the EHRs examined. For example, roughly $80 \%$ of responding students reported that both drug dosage and frequency are important to document. However, the EHR review found that both dosage and frequency were documented in only onethird of the charts (Table 2). This finding indicates a majority of the students missed the opportunity to enhance their pharmacology knowledge and understand patients' health status.

The primary barrier to complete medication documentation cited by these students was patients' provision of inaccurate or missing medication information (Table 1). This problem likely contributes to the finding that approximately one in five records contained medications that were not listed by their correct name. Addressing this problem may be aided by a more intentional approach when interacting with patients. Upon initial phone contact with IUSD clinic staff, patients must be instructed that a complete and accurate list of medications should be brought with them to their first visit to the clinic. Likewise, instructions for new patients on the school's website must clearly provide the reason for a complete medication history and request that patients supply the information upon arrival to their first appointment. This practice should be followed-up with a hard stop that does not allow care to proceed without a complete medical history that includes medication history. Faculty must also assume responsibility for ensuring that a complete and accurate medical history, including medication documentation, is obtained in all student-patient encounters prior to electronic approval of this section of the EHR.

When we chose the individual score components for assessing medication-level \% completeness, a (perhaps subjective) relative importance of each score component was not assigned. However, incorrectly documenting the proper name of a medication was a major contributor to the likelihood of generating incomplete medication documentation. Moreover, nearly one-third of patient EHRs had inconsistencies between patient-reported medications and medical conditions as documented by the students (Table 3). Such inconsistencies clearly raise an important patient safety concern since proper and successful oral health care depends on clinicians' accurate assessment of their patients' overall medical history. It is likely the student clinicians in these cases did not probe further to identify and clarify the source of the inconsistencies.

This study found that greater accuracy and completeness in documenting dental patients' medical histories in the EHR are needed. Therefore, we recommend a multipronged approach for improving medication documentation that involves systems, faculty, students, patients, and curriculum (Figure 3 ). For example, faculty members need to set expectations for what should be included in medication documentation through classroom instruction and clinical training, engagement, and reinforcement. Students must be made aware of expectations, and resources and systems must be in place to facilitate adherence. Systems interventions include clinical policies to optimize staff-patient-student communications as to what is required and why it is important, along with axiUm modifications to facilitate retrieval and recording of drug information. The latter of these systems interventions may include use of an embedded drop-down axiUm menu of commonly used drugs, although, at present, the use of this feature is impractical and may interfere with our pharmacology education objectives. Additionally, the curriculum must emphasize integration of pharmacology and medicine concepts in clinical care, and students should be assessed in this competency during their clinical training.

This study may have limited generalizability because the data were from a single academic dental institution. Another limitation is that the survey data were by self-report. Despite these limitations, this study provides a better understanding of the challenges dental students face in properly documenting patients' medical history, including medications. Moreover, the results revealed an opportunity to enhance pharmacology knowledge and the integration of foundational science with clinical education, while having a positive impact on patient safety and oral health.

\section{Conclusion}

Our survey found that most of the dental students perceived patient medication documentation as important; however, a moderate percentage of the participants did not appreciate the significance of many elements of a medication history. Moreover, the results of the comprehensive and quantitative assessment of the students' actual practices demonstrated a low level of complete and accurate medication 


\section{Systems}

- axiUm modifications to facilitate retrieval of info from drug references

- Communication with patients on importance and necessity of providing current medical and medication information at appointments

- Clinic flow to allow for adequate medical history intake and review

\begin{tabular}{|l|}
\multicolumn{1}{|c|}{ Patients } \\
Bring medications or \\
medication \\
information to dental \\
appointments \\
Provide current \\
medical information \\
and other health \\
care contact \\
information \\
Provide pharmacy \\
information
\end{tabular}

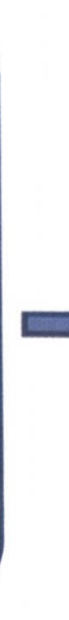

\section{Students \& Faculty}

- Required training on medications/medical history-taking

- Develop and set expectations for student presentations of patients prior to dental care

- Faculty calibration for consistent approach to student presentation of patient information prior to care

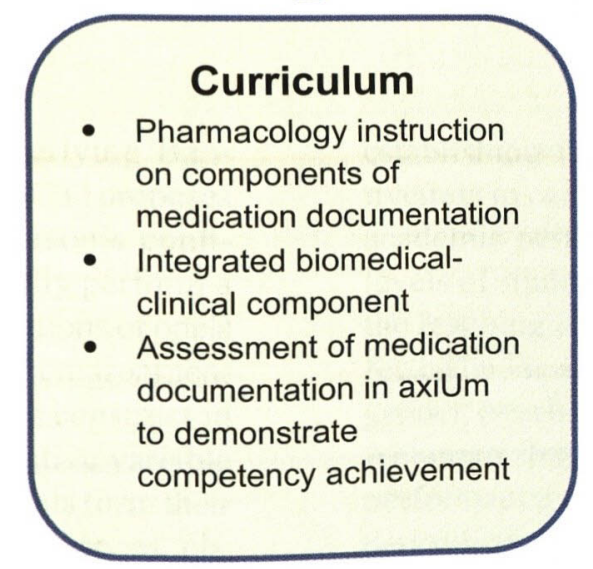

Figure 3. Recommendations to improve dental students' documentation of patient medication in axiUm

documentation. These study findings suggest that improving medical history documentation must be addressed at multiple levels and should include modifications in curriculum, faculty development, and clinical processes and systems.

\section{Acknowledgments}

This project was supported by the Indiana University School of Dentistry's Department of Biomedical Sciences and Comprehensive Care. 


\section{REFERENCES}

1. Becker D. Drug allergies and implications for dental practice. Anesth Prog 2013;60(4):188-97.

2. Bennett J. Preoperative preparation and planning of the oral and maxillofacial surgery patient. In: Todd D, Bennett J, eds. Patient safety. Chicago: Elsevier, 2017:131-3.

3. Centers for Disease Control and Prevention. Therapeutic drug use. At: www.cdc.gov/nchs/fastats/drug-usetherapeutic.htm. Accessed 30 Jan. 2018.

4. Mariotti A. Appendix 4: drug interactions in clinical dentistry. In: Dowd F, Johnson B, Mariotti A, eds. Pharmacology and therapeutics for dentistry. $7^{\text {th }}$ ed. St. Louis: Elsevier, 2016:650-5.

5. Dick G, Lederman B, Hyde S. Oral diseases \& disorders. In: Williams BA, Chang A, Ahalt C, et al., eds. Current diagnosis $\&$ treatment: geriatrics. $2^{\text {nd }}$ ed. New York: McGraw-Hill, 2014:392-8.
6. Gautam M, Shaw DH, Pate TD, Lambert HW. Pharmacology education in North American dental schools: the basic science survey series. J Dent Educ 2013;77(8):1013-21.

7. Gregson K, Romito LM, Garetto LP. Students' attitudes toward integrating problem-based learning into a D.D.S pharmacology curriculum. J Dent Educ 2010;74(5):489-98.

8. Gadbury-Amyot C, Brockman WG. Transition of a traditional pharmacology course for dental students to an online delivery format: a pilot project. J Dent Educ 2011;75(5):633-45.

9. Gregson KS, Romito LM. Using the patient's medication history as a learning tool in clinical pharmacology instruction for dental students. J Dent Educ 2012;76(11):1482-90.

10. Branch-Mays G, Pittenger AL, Williamson K, et al. An interprofessional education and collaborative practice model for dentistry and pharmacy. J Dent Educ 2017;81(12):1413-20.

11. Lygre H, Kjome RLS, Choi H, Stewart AL. Dental providers and pharmacists: a call for enhanced interprofessional collaboration. Int Dent J 2017;67:329-31. 